\title{
Analysis of electrodermal evidence for a para- medullary afferent tract in patients with a transection of the thoracic spinal cord ${ }^{1}$
}

\author{
MARCUS J. FUHRER ${ }^{2}$
}

From the Departments of Rehabilitation and Psychiatry, Baylor College of Medicine, Houston, Texas, U.S.A.

SUMMARY An effort was made to corroborate earlier reports that the central nervous system rostral to a functionally complete transection of the human spinal cord remains responsive to noxious stimulation of sites below the level of the transection. Nine patients were studied who had sustained a functionally complete transection of the thoracic spinal cord between T5 and T11 spinal segments. Noxious electrocutaneous stimulation or intense pressure was applied to a lower extremity while electrodermal activity was recorded concurrently from contralateral palmar sites which were shown to be under normal suprasegmental control. While electrodermal responses were occasionally recorded in the post-stimulus intervals, there was no tendency for these responses to exceed the number or amplitude of responses recorded during stimulus-free control periods. These results were interpreted as suggesting that the few responses observed during the post-stimulus intervals were not evoked by stimulation of the lower extremities and were instead representative of spontaneous electrodermal activity or were related to uncontrolled auditory or visual stimuli accompanying lower extremity stimulation. Additional results highlighted the importance of controlling accessory auditory and visual cues occurring in conjunction with lower extremity stimuli. In some patients with a transection above the sympathetic outflow to the lower extremities, it was shown that electrodermal responses from the plantar aspect of each foot could be elicited reliably by lower extremity stimuli. These results confirmed previously reported evidence that the functionally isolated human spinal cord can reflexly mediate electrodermal responses.

There have been recurring reports that transection of the human spinal cord may not eliminate afferent neural input to the brain from stimulus sites below the level of the lesion. Much of the evidence has depended upon patients' verbal reports of their subjective experience. Interoceptive stimuli resulting from bladder or bowel distension have been reported to produce sensations described as 'unpleasant stretching', 'throbbing', or 'burning in the abdominal or genital regions' (Foerster, 1936; Davis and Martin, 1947; Guttmann and Whitteridge, 1947; Kuhn, 1950; Beneš, 1968). Exteroceptive stimuli may also be effective. Kuhn (1950) describes patients for whom noxious stimulation of skin in the genital

'Supported by Research Grant NS07755-02 from the National Institute of Neurological Disease and Stroke and by Grant RT-4 from the Social and Rehabilitation Service.

'Also at The Texas Institute for Rehabilitation and Research, Texas Medical Center, Houston, Texas 77025, U.S.A., to which address requests for reprints should be sent. region or irritation of a decubitus ulcer on a lower extremity produced a diffuse burning sensation referred to the lower portion of the body.

There have also been efforts to confirm objectively that afferent input originating below the level of a transverse lesion of the spinal cord may effectively bypass the transection (Foerster, 1936; Sourek, 1964, 1965) For this purpose, patients were chosen with a level of injury below the sympathetic outflow to the upper extremities so that peripheral sympathetic activity in these areas remained under normal suprasegmental control. It was then determined whether stimulation applied below the level of the transection was systematically associated with a phasic increase in the peripheral sympathetic activity of the upper extremities. This activity was recorded as either a vasoconstrictive reaction in the arms or as electrodermal responses which reflect a transitory increase of sweat gland activity. Foerster (1936), the first to explore this approach, found that 
the application of extreme pressure to the toes of a patient with a surgically confirmed transection of the thoracic cord resulted in both electrodermal responses and peripheral vasoconstriction in an upper extremity Essentially similar findings were obtained more recently by Sourek (1965). Electrocutaneously stimulating the lower extremities of a patient with a long-standing transection of the cord at T8 was reported to have elicited electrodermal responses from the hand ipsilateral to the stimulus site, though the patient reported no sensation attributable to the stimulus. Four other patients were studied, all of whom showed upper extremity responses to lower extremity stimulation.

To account for the foregoing evidence, Foerster and Sourek posit the existence of a 'paramedullary afferent tract' which receives afferent input from the lumbosacral spinal nerves, courses up the paravertebral chain, and enters the posterior roots at a level rostral to the lesion. As a result, afferent stimulation arising below the level of the lesion is hypothesized to bypass the spinal-cord transection.

In the present investigation, an effort was made to confirm the observations of Foerster and Sourek while adding the procedural controls needed to rule out alternative interpretations of the findings. It was not deemed sufficient to demonstrate merely that electrodermal responses from the upper extremities of such patients can be recorded on occasion following lower extremity stimulation. Instead, an attempt was made to show in addition that upper extremity responses are contingent upon delivery of the stimulus. It was thus necessary to control for the influence of spontaneous electrodermal responses which are known to occur in the absence of discrete exteroceptive stimuli (for example, Johnson, 1963; Bloch, 1965). The possible confounding effects of spontaneous electrodermal activity were taken into account by establishing control periods during which the stimulus to the lower extremity was withheld. The frequency and amplitude of responses observed during poststimulus periods were then compared with the characteristics of responses recorded during control periods. Caution was also taken to eliminate auditory or visual cues associated with delivery of the stimulus. This requirement is particularly important because of the non-specific nature of electrodermal responses-that is, the fact that such responses can be elicited by stimuli in all modalities. To determine the importance of controlling accessory stimuli, a comparison was made of upper extremity responsiveness when an accessory auditory cue was available and when it was eliminated.

In addition to recording from upper extremity sites, the electrodermal activity of the plantar aspect of each foot was also monitored while applying $\bar{z}$ noxious stimuli to lower extremity sites. Of particular interest were results obtained from patients with aō thoracic spinal-cord transection above the sympathetic outflow to the lower extremities. Study of these patients afforded the opportunity to confirmo the earlier results of Fuhrer and Kilbey (1967) whos reported that the functionally isolated human spinal cord can reflexively mediate lower extremity electro dermal responses to noxious cutaneous stimulation applied to sites below the level of the transection.

\section{METHOD}

The nine patients are characterized in the Table. A history and neurological examination revealed that each had sustained a traumatic injury of the spinal cord resultinge in the complete loss of voluntary motor functioning and $\vec{\circ}$ sensation below the level of the lesion. At the time of the study, these individuals were either inpatients on full $\vec{\omega}$ activity schedules or outpatients who had completed ano intensive rehabilitation programme. All were free of urinary tract infections and active decubitus ulcers, ando were not taking drugs with known sympathomimetic or sympatholytic effects. A normal comparison group of sid males and two females between the ages of 18 and 040 was also studied.

\section{TABLE}

CHARACTERISTICS OF THE PATIENTS

\begin{tabular}{|c|c|c|c|c|}
\hline Patient & Sex & $\begin{array}{l}\text { Age } \\
(y r .)\end{array}$ & Level of lesion & $\begin{array}{c}\text { Duration of inju位. } \\
\text { (months) }\end{array}$ \\
\hline $\mathbf{A}$ & $\mathbf{M}$ & 16 & T5/6 & 12 \\
\hline B & $\mathbf{M}$ & 18 & T8 & 26 \\
\hline C & $\mathbf{M}$ & 22 & T5/6 & 18 \\
\hline D & $\mathbf{F}$ & 22 & $\mathrm{~T} 10 / 11$ & 7 \\
\hline $\mathrm{E}$ & $\mathbf{M}$ & 25 & T7 & 54 \\
\hline $\mathbf{F}$ & $\mathbf{M}$ & 17 & T7 & 7 \\
\hline G & $\mathbf{F}$ & 13 & T11 & 16 \\
\hline $\mathbf{H}$ & $\mathbf{M}$ & 18 & T10 & 6 \\
\hline I & $\mathbf{M}$ & 16 & T6/7 & 13 \\
\hline
\end{tabular}

Electrodermal activity was recorded concurrently from two skin sites by means of independent, electronically regulated constant current sources $(15 \mu \mathrm{a})$ and in dependent follower amplifiers with an input impedance? of approximately $100 \mathrm{M} \Omega$. For recording electroderma responses in ohms, the output of each amplifier wasO capacitor-coupled (4 sec time constant) to a two-channel servo recorder (Texas Instruments) or a six-channelo oscillograph (Siemen's Model E). The 2 sq. cm, Ag-AgCl electrodes which were applied to the active skin siteso have been described by Day and Lippitt (1964). These electrodes permitted a current density of $7.5 \mu \mathrm{a} / \mathrm{sq} . \mathrm{cm} N$ Each active site was referenced to a $130 \times 80 \mathrm{~mm}_{\mathrm{r}}$ $\mathrm{Ag}-\mathrm{AgCl}$ electrode.

Electrocutaneous stimuli were provided by the trans former-isolated output of a stimulator (E \& M Instru- 
ment) which supplied a train of $1 \mathrm{msec}$ rectangular pulses at $50 \mathrm{~Hz}$. The $0.5 \mathrm{sec}$ duration of the pulse train was controlled by an electronic timer (Hunter). The $\mathrm{Ag}-\mathrm{AgCl}$ stimulus electrodes were of the same type used for electrodermal recordings. The output current was monitored by a transformer-isolated probe, and read from an oscilloscope (Hewlett-Packard, Model 130B). Binaural audio stimulation was provided by a $2400 \mathrm{~Hz}$ audio oscillator, the output of which was amplified and led into earphones.

A minimum of three sessions on consecutive days was conducted with each patient. To prepare the electrodermal recording sites, the plantar arch of each foot and the hypothenar eminence of each palm were wiped with an acetone-ether solution, the electrodes were filled with gel $(0 \cdot 1 \mathrm{~N} \mathrm{NaCl})$, and a stomeseal ring was used to affix the electrode to the skin. The reference electrode was coated with gel and taped to the lateral aspect of the right lower leg. Stimulating electrodes were affixed with masking tape to the medial plantar aspect of the foot anterior to the electrodermal recording site. The patient lay supine on an examination table, with the recording equipment positioned so that the investigator's movements in operating it were not visible to the patient. Laboratory temperatures averaged $24^{\circ} \mathrm{C}$ (standard deviation $=1.5^{\circ} \mathrm{C}$ ).

During a particular session, continuous records were obtained concurrently from either the pair of palmar sites, the pair of plantar sites, or from a palmar and plantar site which were ipsilateral to the extremity being stimulated. A typical paradigm for a session consisted of a 15 minute period to permit stabilization of skin resistance levels, a six minute recording period while the patient was quiet and relaxed, and several periods during which series of electrocutaneous or pressure stimuli were administered separately to each lower extremity. In some series, a maximum intensity stimulus was established by successively increasing the current until components of the flexor withdrawal reflex of the lower extremities became unacceptably widespread and vigorous or until a maximum current density of $30 \mathrm{~mA} / \mathrm{sq}$. cmı was achieved. This intensity exceeded the level which could be tolerated when sites with preserved sensation were stimulated, and was deemed the highest permissible in order to avoid damage of the skin. In other series, a current level was established which was just below the threshold for movements of the lower extremities, thus eliminating visual cues to the patient associated with delivery of the stimulus. During all periods in which current levels were being established, electrodermal activity was monitored continuously in order to determine the threshold for responses from particular sites. After setting the current level, 10 or more stimuli were applied to the foot at approximately 20 second intervals. Stimulus presentations were interspersed with control periods during which the procedure for administering a stimulus was executed with an open circuit to the patient. Various movements of the lower extremities were carefully observed and coded as to type and intensity on the record.

In at least one session with each patient, electrodermal activity was recorded bilaterally from the palms while electrocutaneously stimulating lower extremity sites other than the plantar surfaces. The additional sites were both below and above the knees and included the buttock and thigh regions. These sites were also submitted to intense manual pressure by tightly squeezing both the skin and underlying musculature or the skin alone. The application of pressure was recorded on an event channel of the recorder by means of a microswitch taped to the skin. During this procedure, a curtain was used to obscure the patient's view of his lower body.

For four of the patients, a part of one session was devoted to determining how palmar electrodermal responsiveness was affected by associating an auditory cue with stimulation of the foot. For this purpose, a relay which produced an audible click when the shock was initiated was switched into the shock circuit. The patient was informed that when a click was heard, a shock was being delivered to the foot. In addition, the patient was requested to attend to each click. In the subsequent series, the shock and click were sometimes presented together, and on other occasions, either the click or the shock was presented separately.

During the initial session with each patient, a test was conducted to determine how the electrodermal responsiveness of sites on each of the four extremities compared with the responsiveness of neurologically intact individuals. Both a noxious electrocutaneous stimulus and a mild, $50 \mathrm{db}$ auditory stimulus were used. The electrocutaneous stimulus consisted of a 0.5 second train of $\mathbf{5 0}$ $\mathrm{Hz}$ pulses applied to a normally sensitive area of the patient's neck. The intensity of the stimulus was successively increased to a level described by the patient as painful, but tolerable. When this level was reached, at least eight stimuli were applied while recording concurrently from the left palm and the plantar arch of the left foot. This procedure was then repeated for ipsilateral sites on the right side of the body. Both combinations of recording sites were also monitored while 0.5 second, $50 \mathrm{db}$ tones were presented through earphones. There were at least six repetitions of the tone, separated by 10 to 30 second intervals. The procedures involving tones and tolerance-level shocks were also conducted with the normal subjects.

\section{RESULTS}

It was confirmed for each patient that the responsiveness of palmar recording sites to noxious cutaneous stimulation and to the auditory stimuli was comparable with that of normal individuals. For these determinations, a palmar response was defined as a transitory decrease in resistance of $100 \Omega$ or more, occurring with a latency of 1 to 3 seconds after onset of the stimulus. Responses from plantar sites were scored in the interval 2 to 4 seconds from stimulus onset. The portion of the record in Fig. 1 typifies the reactions of neurologically intact individuals to the painful stimulus applied to the neck while recordings were obtained from the left palm and the sole of the left foot. In this record, an electrodermal 


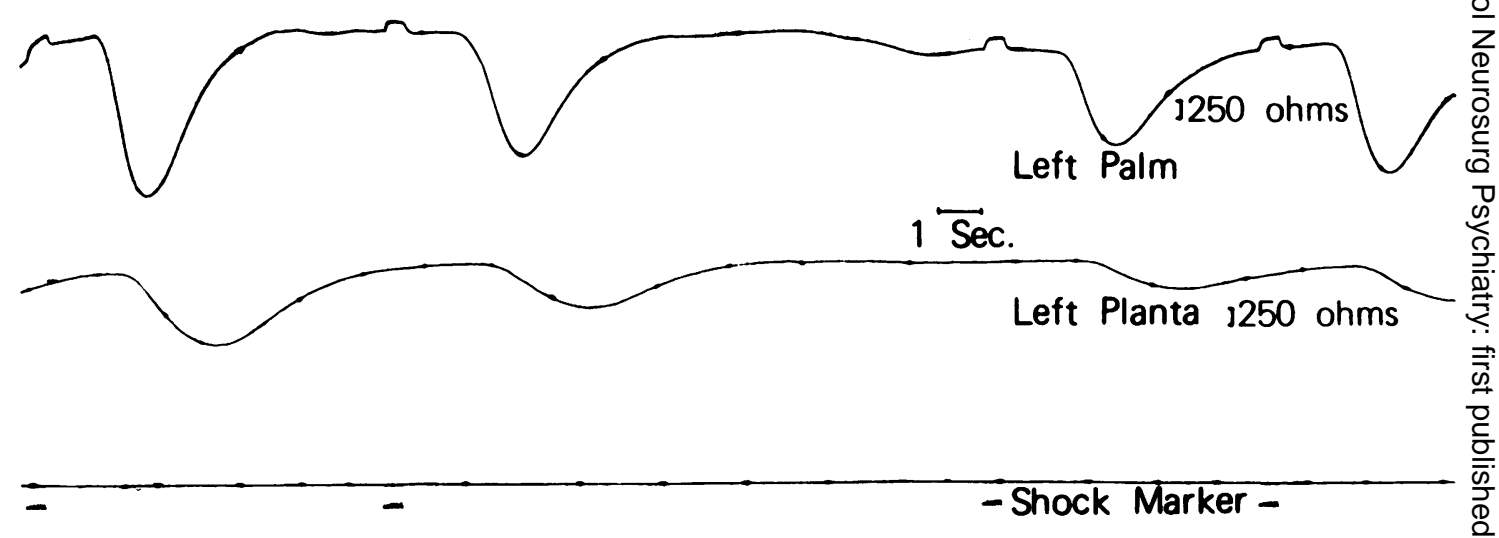

FIG. 1. Electrodermal responses of a normal subject recorded from the left palm and the plantar arch of the left foot.œ Deflections of the shock marker and a concomitant artefact in the palmar electrodermal trace indicate points at which $\overrightarrow{0}$ noxious, electrocutaneous stimulation of 0.5 second duration was applied to the lateral aspect of the neck.

response consists of a downward deflection of the trace indicating a transitory decrease of skin resistance. A deflection of the shock marker and a concomitant artefact in the palmar electrodermal trace indicate when the shock was applied. Approximately 1.6 seconds after onset of the stimulus, a response was recorded from the palm, and after a somewhat longer delay, from the left plantar site as well. Other recordings obtained concurrently from the two palmar sites or from the sole of each foot revealed a similar picture, except that the latency of responses from contralateral sites was indistinguishable. Auditory stimuli were also effective in eliciting this pattern of activity, the chief differences being that responses were of generally smaller amplitude, and $\omega$ they tended to decrease in amplitude systematicalt $\stackrel{+}{\omega}$ with repetition of the stimulus. Among the normâlin subjects, the average latency of palmar responses $\mathbb{R}_{-}^{\infty}$ both tones and shocks ranged between 1.5 and 201 응 seconds. The average latency of plantar responseswas in the range of $2 \cdot 2$ to $2 \cdot 8$ seconds.

The portion of the record shown in Fig. 2 wogs obtained from patient $C$. The upper electrodermil trace exemplifies the responsiveness of patient palmar sites to stimulation of the neck. Like the normals, all patients responded at both palmar sites

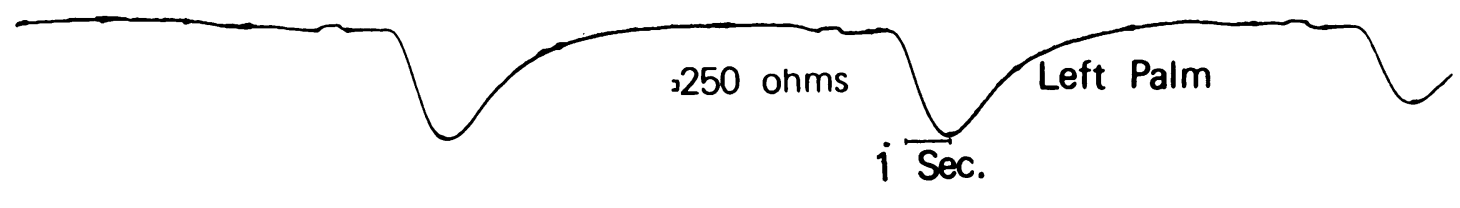

J 250 ohms

Left Planta

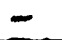

FIG. 2. Electrodermal activity of the left palm and the plantar arch aspect of the left foot for patient C. Noxious, electro- $-{ }^{2}$ cutaneous stimulation of 0.5 second duration was applied to the lateral aspect of the neck at points indicated by a deflection 0 of the shock marker and a concomitant artefact in the palmar electrodermal trace. Electrodermal responses were onlyN elicited from the palmar site. 
to a minimum of eight consecutive presentations of the shock and six presentations of the tones. Neither the amplitude nor the latency of patients' palmar responses differed from the values obtained for normals.

The reactivity of plantar recording sites depended upon the level of the spinal-cord injury. Consistent responding to noxious stimulation was obtained from both plantar sites for patients $D, G$, and $H$. These patients had a level of injury no higher than T10 spinal segment. Plantar responding could not be elicited from the remaining patients who had sustained a spinal-cord injury at T8 segment or above. This finding is exemplified in the lower electrodermal trace in Fig. 2. Application of shocks to the neck of patient $\mathrm{C}$ yielded no responses from the left plantar site, though high amplitude responses were recorded from the left palmar site.

Evidence for a relationship between lower extremity stimulation and upper extremity electrodermal responding was sought in a variety of recordings obtained from each patient. For both electrocutaneous and pressure stimuli, recordings were available in which each lower extremity was stimulated separately while concurrently recording from contralateral palmar sites or from palmar and plantar sites ipsilateral to the extremity being stimulated. A palmar electrodermal response was tallied and its amplitude measured in ohms if it originated 1 to 3 seconds after onset of the stimulus. The same latency range was used to score responses observed during stimulus-free control periods which were interspersed among stimuli. If electrodermal responses were recorded in six or more post-stimulus intervals, the Wilcoxon test (Siegel, 1956) was computed to determine if responses in the poststimulus intervals differed significantly in frequency and amplitude from responses recorded during control periods.

The recordings were predominantly characterized by an absence of palmar electrodermal responses in both the post-stimulus and control periods, regardless of which foot was being stimulated and irrespective of the type or intensity of stimulation. A typical outcome, derived from the data for patient $\mathrm{B}$, is shown in Fig. 3. During this portion of the record, the electrodermal activity of the right palm and the sole of the right foot were recorded while maximum intensity shocks of a current density of $30 \mathrm{~mA} / \mathrm{sq} \mathrm{cm}$ were being applied to the plantar aspect of the right foot. Points at which the stimulus was applied are discernible by noting a deflection of the shock marker and a concomitant artefact in the plantar electrodermal trace. Designation of a control period is indicated by a deflection of the shock marker which is not associated with a shock artefact in the electrodermal trace. During this recording, shocks were not followed by electrodermal responses from the right palmar site, nor were lower extremity movements elicited by the stimulus. Responses obtained from the right plantar site are discussed below.

With one exception which will be discussed in detail, palmar responses after either shock or pressure stimuli were recorded in no more than $25 \%$ of the post-stimulus intervals, the minimum number of stimulus repetitions being 10. The responses which were observed did not differ remarkably in frequency and amplitude from responses recorded during control periods. The effects were also ex-

\section{Right Palm ]250 ohms}



\footnotetext{
Shock Marker -

FIG. 3. Electrodermal activity of the right palm and the plantar aspect of the right foot for patient B. A O.5 second pulse train with an intensity of $30 \mathrm{~mA} / \mathrm{sq} . \mathrm{cm}$ was applied to the medial plantar aspect of the right foot at points at which a deflection of the shock marker occurs in conjunction with an artefact in the plantar electrodermal trace. The deflection of the shock marker which is unaccompanied by a shock artefact indicates a control period in which the pulse train was withheld. Actual delivery of the stimulus resulted only in responses from the plantar recording site.
} 
plored of scoring responses which occurred as late as 8 seconds after onset of the stimulus. This resulted in slightly increasing the number of poststimulus intervals in which a response was observed. However, similarly extending the duration of the control periods resulted in a commensurate increase in the number of these periods in which a response occurred.

The only case of consistent palmar responding to lower extremity stimulation occurred in one stimulus series with patient I. In this series, shocks to the left plantar surface consistently elicited flexor withdrawal movements of the left leg, including brisk dorsiflexion of the toes and ankle and slight flexion of the left knee. In this situation, 14 of the 16 stimulus repetitions resulted in bilaterally recorded palmar responses which differed significantly in amplitude from responses recorded during control periods $(T=3, P<0.01)$. To assess the possibility that these results were due to the patient visually attending to the leg movements, a screen was positioned so as to obscure the patient's view of his lower extremities. Under these conditions, palmar responses were recorded after only two of the 15 stimulus presentations, with three responses being observed during control periods. Flexor activity similar to that described for patient I was also elicited during stimulus series for patients $B, D, F$, and $H$, though the incidence of palmar responses was no greater than observed with stimulus intensities below the threshold for lower extremity movements.

The influence of accessory stimuli on palmar responsiveness was discernible in the data of four patients who were submitted to the additional condi- $\underline{0}$ tion in which an audible click sometimes occurred in $\underset{\mathbb{O}}{Z}$

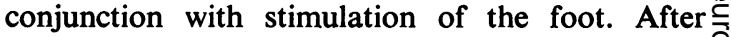
calling attention to the presence of the click, bilateral of palmar responses were recorded after the initial presentations of the stimulus. Responses to suc- $D$ cessive clicks tended to decrease in amplitude, and $\stackrel{0}{-}$ became intermittent after approximately the tenth $\stackrel{5}{\mathrm{~S}}$. repetition. Particularly important was the observation that responding occurred even when shock to?. the foot was omitted and only the click was pre- $\vec{F}$ sented. Conversely, applying the stimulus to the foot $\frac{\text { ? }}{7}$ but omitting the click was associated with an absence of palmar responding. Figure 4 contains an $\frac{\bar{\sigma}}{\bar{D}}$ example of results obtained for patient A while $0.5 \frac{\pi}{\widetilde{\alpha}}$ second pulse trains with an intensity of $30 \mathrm{~mA} / \stackrel{\circ}{\Omega}$ sq. $\mathrm{cm}$ were being applied to the right plantar sur- $\omega$ face. Presentations of the click resulted in $\vec{\circ}$ bilateral palmar responses, regardless of whether the click was accompanied by stimulation of the foot. $\vec{\omega}$ No responses occurred when the foot was stimulated but the click was omitted.

For some patients, it was observed that the distal $\stackrel{i}{\omega}$ segment of the transected spinal cord can mediate $\stackrel{+}{\omega}$ plantar electrodermal responses to lower extremi stimulation. The pertinent data were contained in tige records of the patients with a transection above T segment. As noted above, evidence was available that the plantar electrodermal activity of these six $\bigodot_{C}$ patients was independent of suprasegmental controp. For patients $\mathrm{B}, \mathrm{C}$, and $\mathrm{F}$, plantar responses were consistently observed after electrocutaneous stimuk tion of the sole of the foot. Examples of responses.
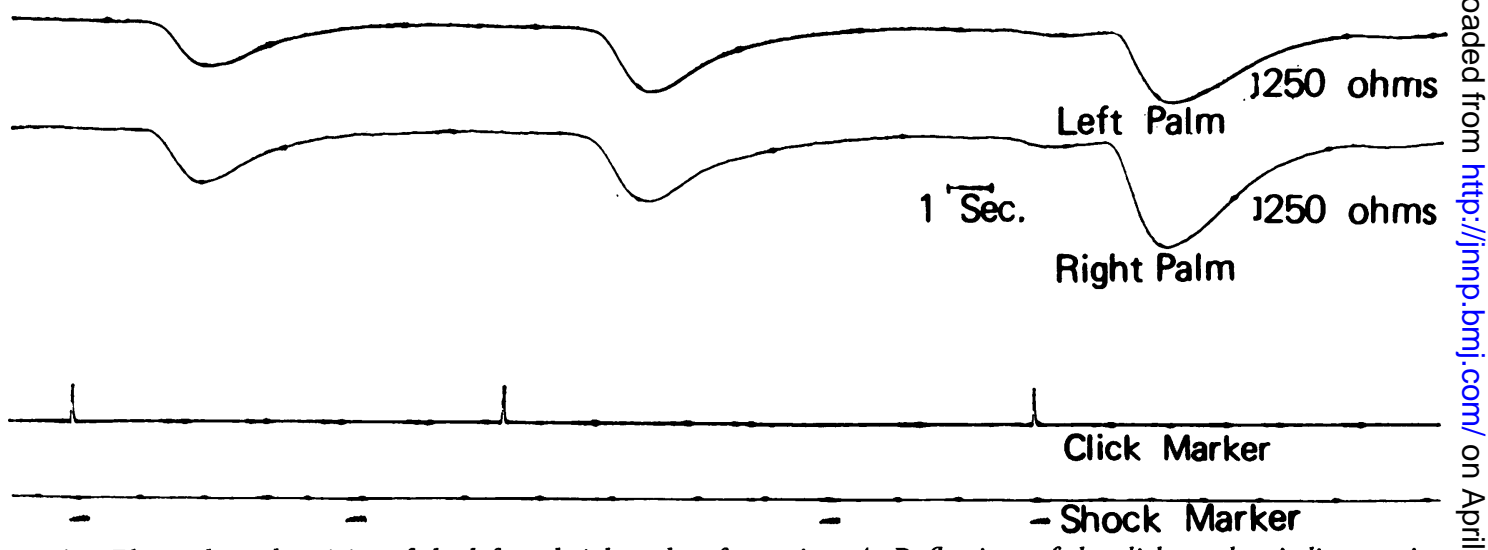

FIG. 4. Electrodermal activity of the left and right palms for patient A. Deflections of the click marker indicate points tt which an audible click was presented. Deflections of the shock marker indicate occasions when a 0.5 second pulse train 5 with an intensity of $30 \mathrm{~mA} / \mathrm{sq}$. cm was applied to the medial plantar aspect of the right foot. Bilateral palmar responses $\mathrm{N}$ were elicited by a joint presentation of the click and the shock, as well as by presentations of the click alone. No responses $\mathrm{N}$ were evoked by presenting the shock alone. 
elicited from patient B are contained in the lower electrodermal trace in Fig. 3. During this recording, stimulating and recording electrodes were located on the sole of the right foot, and a site on the palm of the right hand was also being monitored. On other occasions with this patient when contralateral plantar sites were being monitored concurrently, bilateral responding was observed. Responses from the foot being stimulated appeared at a stimulus intensity substantially lower than that required for responses from the contralateral foot. At a stimulus intensity which was suprathreshold for responding from both sites, the amplitude of responses from the stimulated foot was consistently higher than the amplitude of responses from the opposite site. When the stimulus site was shifted to the other foot, the relative amplitude of the plantar responses was reversed. For patients $\mathrm{C}$ and $\mathrm{F}$, responses were elicited reliably only from the foot being stimulated. Plantar responses could be elicited only at relatively high current levels, $10 \mathrm{~mA} / \mathrm{sq} . \mathrm{cm}$ being the lowest current density required in the multiple series conducted with each of the three patients. The amplitude of responses to suprathreshold stimuli increased systematically as the intensity of the stimulus was raised. Even at maximum stimulus intensities, responses from plantar sites were of lower amplitude than those recorded from palmar sites to auditory stimuli or to electrocutaneous stimulation of the neck.

\section{DISCUSSION}

The results of this study do not support the contention that peripheral sympathetic responses from sites above the level of a transverse lesion of the human spinal cord can be elicited by noxiously stimulating skin sites below the lesion. Consistently negative evidence was obtained, despite the presence of several factors which should have favoured demonstrating the phenomenon. A deliberate effort was made to select patients with transverse lesions at different levels of the thoracic spinal cord. It was shown for each patient that electrodermal recording sites on both upper extremities were normally responsive to stimuli applied above the level of the lesion. Multiple sites on the lower extremities were stimulated using both electrical and pressure stimuli. The intensity of electrocutaneous stimulation was varied over a wide range, with the maximum intensity being substantially stronger than could be tolerated by neurologically intact individuals.

In accounting for the discrepancy between the present results and those of Foerster (1936) and Sourek $(1964,1965)$, it may be noted that several conditions were imposed upon the present study which were lacking in the earlier investigations. Of probable significance was the effort to control the influence of spontaneous electrodermal responses from upper extremity sites. It is well established in normal individuals that electrodermal responses can be recorded in the absence of specific exteroceptive stimuli, and that the frequency of these responses is related to the individual's state of alertness (Burch and Greiner, 1960; Bloch, 1965). In the present study, the incidence of spontaneous electrodermal responses and their amplitude were sampled during stimulus-free intervals which were interspersed between presentations of lower extremity stimuli. It was repeatedly found that the number and amplitude of the occasional responses recorded in post-stimulus periods were not distinguishable from the characteristics of responses obtained during the stimulus-free intervals.

The procedures of the current study were also influenced by the knowledge that electrodermal responses may be elicited by stimuli in all sense modalities. Consequently, every effort was made to eliminate visual or auditory cues which might accompany lower extremity stimulation. Under these conditions, systematic upper extremity responding was not observed. The importance of controlling accessory cues was readily demonstrated by adding an audible click which the patient knew was associated with shock to the foot. With this modification, upper extremity responses were consistently observed in the post-stimulus intervals, though the responses tended to become extinguished with frequent repetition of the stimulus. There was also some evidence that palmar electrodermal responses could be elicited by visual cues accompanying flexor withdrawal movements of the lower extremity. The electrodermal responses dropped out when the patient's view of his lower limbs was obstructed.

The present findings are thus consistent with the conventional view that transection of the spinal cord blocks transmission of cutaneous input to the brain from skin sites innervated below the level of the lesion. These findings, however, by no means rule out the possibility that other kinds of afferent input, especially from the viscera, may bypass a transverse lesion of the cord. Studies of the anaesthetized cat suggest that stimulation of splanchnic nerves can be conducted up the paravertebral sympathetic chain and enter the spinal cord via the posterior roots from T3 to L1 (Bain, Irving, and McSwiney, 1935). Cooper and Kerslake (1955) have also shown in anaesthetized human subjects that vasoconstriction in the hand can be evoked by stimulating the lumbar sympathetic chain. Furthermore, clinical observations of paraplegic patients indicate that a substantial proportion of these individuals complain 
of diffuse, sometimes painful sensations in the lower body which are associated with distension of the bladder or bowels(Foerster, 1936; Davis and Martin, 1947; Guttmann and Whitteridge, 1947; Kuhn, 1950; Beneš, 1968). Taken together, these types of evidence suggest the potential fruitfulness of further studies along the present lines using phasic stimulation of the viscera.

By monitoring the electrodermal activity of plantar sites, it was possible to confirm the results of Fuhrer and Kilbey (1967) who found that the distal portion of the transected spinal cord can mediate electrodermal responses to noxious stimulation of the lower extremities. This aspect of the study focused upon patients whose injuries were above T9 segment since sympathetic motoneurones supplying the legs originate no higher than this level (Boshes, 1962). It was confirmed that the electrodermal activity of plantar sites is independent of suprasegmental control in such patients, since a painful stimulus applied above the level of the transection was not effective in eliciting plantar responses. As reported in the earlier study, shocks applied to the sole of the foot were not effective in eliciting plantar responses from all appropriate patients. This was probably due in part to limits placed on the maximum stimulus intensity so that damage to the skin could be avoided. This conjecture is consistent with the findings that successful evocation of responses required a relatively intense stimulus, and that once the stimulus became suprathreshold, the amplitude of responses was positively related to the strength of the stimulus. As was found earlier, the responsiveness of plantar sites depended upon the laterality of the stimulus. Response thresholds were lower and the amplitude of responses was greater for the recording site on the same foot which was being stimulated.

\section{REFERENCES}

Bain, W. A., Irving, J. T., and McSwiney, B. A. (1935). The afferent fibres from the abdomen in the splanchnic nerves J. Physiol. (Lond.), 80, 323-333.

Bloch, V. (1965). Le contrôle central de l'activité électro dermale. J. Physiol. (Paris), 57, Suppl. 13, 1-132.

Boshes, B. (1962). Trauma to the spinal cord. Clinicat Neurology, 3, 1639-1678. Edited by A. B. Baker. Hoeber? Medical: New York.

Burch, N. R., and Greiner, T. H. (1960). A bioelectric scale of human alertness: Concurrent recording of the EEG: and GSR. Psychiat. Res. Rep. Amer. psychiat. Ass., No. 12

Beneš, V. (1968). Spinal Cord Injury. Baillière, Tindall, an Cassell: London.

Cooper, K. E., and Kerslake, D. McK. (1955). Vasoconstric tion in the hand during electrical stimulation of the $\overrightarrow{\widetilde{D}}$ lumbar sympathetic chain in man. J. Physiol. (Lond.), 127, 134-142.

Davis, L., and Martin, J. (1947). Studies upon spinal cord injuries. II. The nature and treatment of pain.J. Neurosurg? 4, 483-491.

Day, J. L., and Lippitt, M. W. (1964). A long-term electroded system for electrocardiography and impedance pneumography. Psychophysiology, 1, 174-182.

Foerster, O. (1936). Symptomatologie der Erkrankungen des Rückenmarks und seiner Wurzeln. Handbuch der Neuro logie, 5, 1-403. Edited by $O$. Bumke and $O$. Foerster Springer: Berlin.

Fuhrer, M. J., and Kilbey, M. (1967). Effects of spinal-ce्fid transections on electrodermal activity in man. Psycho physiology, 4, 176-186.

Guttmann, L., and Whitteridge, D. (1947). Effects of blad@er distension on autonomic mechanisms after spinal cord injuries. Brain, 70, 361-404.

Johnston, L. C. (1963). Some attributes of spontane autonomic activity. J. comp. physiol. Psychol., 56, 415-4\$2

Kuhn, R. A. (1950). Functional capacity of the isolate human spinal cord. Brain, 73, 1-51.

Siegel, S. (1956). Non-parametric Statistics for the Behaviorat Sciences. McGraw-Hill: New York.

Sourek, K. (1964). Die bedeutung des galvanischen hautre flexes für die lokalisation von läsionen im nervensystem de menschen. Acta Neurochir., 11, 518-529.

Sourek, K. (1965). The Nervous Control of Skin Potential: in Man. Czechoslovak Academy of Sciences: Prague. 